\title{
MIXINGS, LIFETIMES, SPECTROSCOPY AND PRODUCTION OF HEAVY FLAVOR AT THE TEVATRON
}

\author{
K. T. PITTS \\ University of Illinois, Department of Physics, 1110 West Green St., Urbana, IL 61801, USA \\ E-mail: kpitts@uiuc.edu
}

\begin{abstract}
The Fermilab Tevatron offers unique opportunities to perform measurements of the heavier $B$ hadrons that are not accessible at the $\Upsilon(4 S)$ resonance. In this summary, we describe some recent heavy flavor results from the DØ and
\end{abstract} $\mathrm{CDF}$ collaborations and discuss prospects for future measurements.

\section{Introduction}

In the 1980's and 1990's, the complementary $b$ physics programs of CLEO, ARGUS, the LEP experiments, SLD, DØ and CDF began to make significant contributions to our understanding of the production and decay of $B$ hadrons. With the successful turnon of the BaBar and Belle experiments in the last few years, many experimental measurements in the $b$ sector have achieved impressive precision.

A number of questions remain, and it will again take an effort of complementary measurements to make further progress on our understanding of the $b$ system. The Fermilab Tevatron, along with the upgraded CDF and DØ detectors, offers a unique opportunity to study heavy flavor production and decay. In many cases, the measurements that can be performed at the Tevatron are complementary to those performed at the $e^{+} e^{-} B$-factories.

In this document, we summarize some of the recent experimental progress in the measurements of $B$ lifetimes, spectroscopy, mixing, and heavy flavor production at the Tevatron. Since the details of many of these analyses are presented in other publications, we will attempt to include some background information in this summary that the reader might not find elsewhere.

\section{Overview: $B$ Physics at the Tevatron}

In $p \bar{p}$ collisions at $\sqrt{s}=1.96 \mathrm{TeV}$, the $b \bar{b}$ cross section is large $\mathcal{O}(50 \mu b)$, yet it is only about $1 / 1000^{\text {th }}$ the total inelastic $p \bar{p}$ cross section. At a typical Tevatron instantaneous luminosity of $\mathcal{L}=4 \times 10^{31} \mathrm{~cm}^{-2} \mathrm{~s}^{-1}$, we have a $b \bar{b}$ production rate of $\sim 2 \mathrm{kHz}$ compared to an inelastic scattering rate of $\sim 2 \mathrm{MHz}$.

The $b \bar{b}$ quarks are produced by the strong interaction, which preserves "bottomness", therefore they
Table 1. $B$ mesons and baryons. This is an incomplete list, as there are excited states of the mesons and baryons (e.g. $\left.B^{* 0}\right)$. Also, a large number of $b$-baryon states $\left(e . g . \Sigma_{b}^{-}=\mid d d b>\right)$ and bottomonium states $\left(e . g . \eta_{b}, \chi_{b}\right)$ are not listed.

\begin{tabular}{lll} 
Name & $\bar{b}$ hadron & $b$ hadron \\
\hline charged $B$ meson & $B^{+}=\mid \bar{b} u>$ & $B^{-}=\mid b \bar{u}>$ \\
neutral $B$ meson & $B^{0}=\mid \bar{b} d>$ & $\overline{B^{0}}=\mid b \bar{d}>$ \\
$B_{s}(B$-sub- $s$ ) meson & $B_{s}^{0}=\mid \bar{b} s>$ & $B_{s}^{0}=\mid b \bar{s}>$ \\
$B_{c}(B$-sub- $c)$ meson & $B_{c}^{+}=\mid \bar{b} c>$ & $B_{c}^{-}=\mid b \bar{c}>$ \\
$\Lambda_{b}$ (Lambda- $\left.b\right)$ & $\overline{\Lambda_{b}}=\left|\bar{u} \overline{d b}>\quad \Lambda_{b}=\right| u d b>$ \\
$\Upsilon$ (Upsilon) & \multicolumn{2}{c}{$\Upsilon=\mid \bar{b} b>$} \\
\hline
\end{tabular}

are always produced in pairs. ${ }^{a}$ Unlike $e^{+} e^{-}$collisions on the $\Upsilon(4 S)$ resonance, the high energy collisions access all angular momentum states, so the $b$ and $\bar{b}$ are produced incoherently. As a consequence, lifetime, mixing and $C P$-violation measurements can be performed by reconstructing a single $B$ hadron in an event. The produced $b$ quarks can fragment into all possible species of $B$ hadrons, including $B_{s}, B_{c}$ and $b$-baryons. Table 1 lists the most common species of $B$ hadrons, all of which are accessible at the Tevatron.

The transverse momentum $\left(p_{T}\right)$ spectrum for the produced $B$ hadrons is a steeply falling distribution, which means that most of the $B$ hadrons have very low transverse momentum. For example, a fully reconstructed sample of $B \rightarrow J / \psi K$ decays has an average $B$ meson $p_{T}$ of about $10 \mathrm{GeV} / c$. As a consequence, the tracks from these decays are typically quite soft, often having $p_{T}<1 \mathrm{GeV} / c$. One of the experimental limitations in reconstructing these modes is the ability to find charged tracks at very low momentum. $B$ hadrons with low transverse momentum

${ }^{a}$ It is possible to produce $b$ 's singly through weak decays such as $W^{-} \rightarrow \bar{c} b$ and $W^{-} \rightarrow \bar{t} b$. The cross sections for these processes are several orders of magnitude below the cross section for direct $b \bar{b}$ production by the strong interaction. 
do not necessarily have low total momentum. Quite frequently, the $B$ mesons have very large longitudinal momentum (the momentum component along the beam axis.) These $B$ hadrons are boosted along the beam axis and are consequently outside the acceptance of the detector.

To reconstruct the $B$ hadrons that do fall within the detector acceptance, the experiments need excellent tracking that extends down to low transverse momentum, excellent vertex detection to identify the long-lived hadrons containing heavy flavor, and highrate trigger and data acquisition systems to handle the high rates associated with this physics. In the following section, we outline some of the relevant aspects of the Tevatron detectors.

\section{The CDF and DØ Detectors}

The CDF and D $\varnothing$ detectors are both axially symmetric detectors that cover about $98 \%$ of the full $4 \pi$ solid angle around the proton-antiproton interaction point. For Tevatron Run II, both experiments have axial solenoidal magnetic fields, central tracking, and silicon microvertex detectors. Additional details about the experiments can be found elsewhere. ${ }^{1,2}$ The strengths of the detectors are somewhat complementary to one another and are discussed briefly below.

\section{$3.1 D \varnothing$}

The $\mathrm{D} \varnothing$ tracking volume features a $2 \mathrm{~T}$ solenoid magnet surrounding a scintillating fiber central tracker that covers the region $|\eta| \leq 1.7$, where $\eta$ is the pseudorapidity, $\eta=-\ln (\tan (\theta / 2))$, and $\theta$ the polar angle measured from the beamline. The $\mathrm{D} \varnothing$ silicon detector has a barrel geometry interspersed with disk detectors which extends the forward tracking to $|\eta| \leq 3$. In addition, the $\mathrm{D} \varnothing$ muon system covers $|\eta| \leq 2$ and the uranium/liquid-argon calorimeter has very good energy resolution for electron, photon and hadronic jet energy measurements.

\section{2 $C D F$}

The CDF detector features a $1.4 \mathrm{~T}$ solenoid surrounding a silicon microvertex detector and gas-wire drift chamber. The CDF spectrometer has excellent mass resolution. These properties, combined with muon detectors and calorimeters, allow for excellent muon and electron identification, as well as precise tracking and vertex detection for $B$ physics.

\subsection{Triggering}

Both experiments exploit heavy flavor decays which have leptons in the final state. Identification of dimuon events down to very low momentum is possible, allowing for efficient $J / \psi \rightarrow \mu^{+} \mu^{-}$triggers. As a consequence, both experiments are able to trigger upon the $J / \psi$ decay, and then fully reconstruct decay modes such as $B_{s}^{0} \rightarrow J / \psi \phi$, with $\phi \rightarrow K^{+} K^{-}$. Triggering on dielectrons to isolate $J / \psi \rightarrow e^{+} e^{-}$decays is also possible, although at low momentum the backgrounds become more problematic.

$\mathrm{CDF}$ has implemented a $J / \psi \rightarrow e^{+} e^{-}$trigger requiring each electron have $p_{T}>2 \mathrm{GeV} / c$. Because the triggering and selection cuts required to reduce background are more stringent than they are for the dimuon mode, the yield for the $J / \psi \rightarrow e^{+} e^{-}$ mode is about $1 / 10^{\text {th }}$ the yield for the dimuon mode. However, since selection criteria isolate a dielectron mode is at higher momentum then the dimuon mode, the $B$ purity in the $J / \psi \rightarrow e^{+} e^{-}$channel is higher. The analyses shown here use only the dimuon mode, although future analyses will supplement the signal sample with the dielectron mode.

Both experiments also have inclusive lepton triggers designed to accept semileptonic $B \rightarrow \ell \nu_{\ell} X$ decays. D $\varnothing$ has an inclusive muon trigger with excellent acceptance, allowing them to accumulate very large samples of semileptonic decays. The CDF semileptonic triggers require an additional displaced track associated with the lepton, providing cleaner samples with a smaller yields.

New to the CDF detector is the ability to select events based upon track impact parameter. The CDF Silicon Vertex Tracker (SVT) operates as part of the Level 2 trigger system. Tracks identified by the eXtremely Fast Tracker (XFT) are passed to the SVT, which appends silicon hits to the tracks to measure the impact parameter of each track. With the high trigger rate, it is very challenging to extract the data from the silicon detector and perform pattern recognition quickly. The SVT takes on average $25 \mu \mathrm{s}$ per event to extract the data from the silicon, perform silicon clustering and track fitting. As shown in Fig. 1, the impact parameter resolution for tracks with $p_{T}>2 \mathrm{GeV} / c$ is $47 \mu \mathrm{m}$, which is a combination 


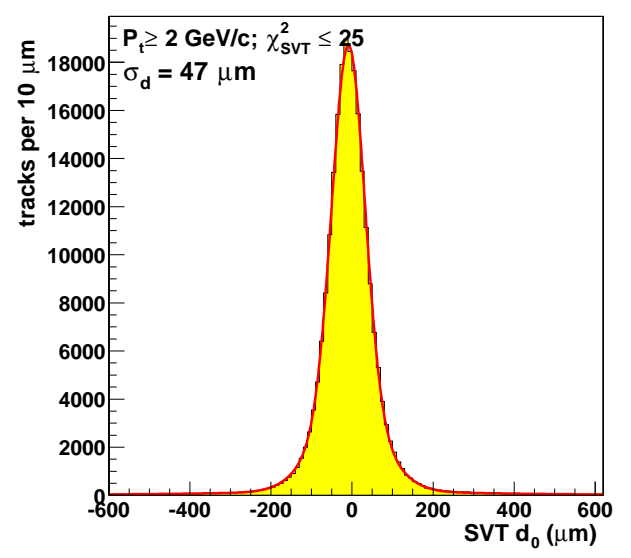

Figure 1. Resolution for the CDF Silicon Vertex Tracker (SVT.) The SVT performs axial tracking in the trigger using the silicon microvertex detector. This plot shows the impact parameter resolution for a sample of tracks. The resolution is $47 \mu \mathrm{m}$. The typical heavy flavor trigger requires two tracks with impact parameter greater than $120 \mu \mathrm{m}$, which has a rejection factor that is 250 times greater than simply demanding charged tracks.

of the primary beam spot size $(30 \mu m)$ and the resolution of the device $(35 \mu \mathrm{m}$.) The CDF SVT has already shown that it will provide a number of new modes in both bottom and charm physics that were previously not accessible. $\mathrm{D} \varnothing$ is currently commissioning a displaced track trigger as well.

\section{Heavy Flavor Yields}

As mentioned in the previous section, the $b \bar{b}$ production cross section is very large at the Tevatron. Although triggering and reconstruction is very challenging, large samples can indeed be acquired. Table 2 shows approximate yields for several different modes at the Tevatron. ${ }^{b}$ As of this writing, each experiment has logged approximately $\mathcal{L}=200 \mathrm{pb}^{-1}$ of integrated luminosity. The data sample is expected to more than double during the 2004 running period.

\section{Prompt Charm Cross Section}

Previously published measurements of the $b$ production cross section at the Tevatron have consistently been significantly higher than the Next-to-Leading Order QCD predictions. Although there has been

\footnotetext{
${ }^{b}$ Throughout this note, we will write charge specific decay modes for clarity. All analyses presented here include the charge-conjugate modes.
}

Table 2. Tevatron yields for various fully- and partiallyreconstructed heavy flavor modes. The column labeled "trigger" lists the primary signature utilized to select the events. The trigger type $d_{0}$ is referring to the impact parameter described in the text. This list is not meant to be exhaustive, only to give the reader a feel for the sample sizes for heavy flavor analyses at the Tevatron. A number of $J / \psi$, semileptonic and all-hadronic decay modes are not included in this table.

\begin{tabular}{cccc}
\hline Hadron & Decay mode & Trigger & Yield per $\mathrm{pb}^{-1}$ \\
\hline$D^{0}$ & $K^{-} \pi^{+}$ & $d_{0}$ & 6000 \\
$D^{+}$ & $K^{-} \pi^{+} \pi^{+}$ & $d_{0}$ & 5000 \\
$B^{-}$ & $D^{0} \pi^{-}$ & $d_{0}$ & 16 \\
$B_{s}^{0}$ & $D_{s}^{-} \pi^{+}$ & $d_{0}$ & 1 \\
$B_{s}^{0}$ & $K^{+} K^{-}$ & $d_{0}$ & 1.5 \\
$J / \psi$ & $\mu^{+} \mu^{-}$ & dimuon & 7000 \\
$B^{+}$ & $J / \psi K^{+}$ & dimuon & 11 \\
$B_{s}$ & $J / \psi \phi$ & dimuon & 1 \\
$\Lambda_{b}$ & $J / \psi \Lambda$ & dimuon & 0.7 \\
$B$ & $D \ell \nu$ & single lepton & 400 \\
$\Lambda_{b}$ & $\Lambda_{c} \ell \nu$ & single lepton & 10 \\
\hline
\end{tabular}

theoretical activity in this arena and the level of the discrepancy has been reduced, it is not yet clear that the entire scope of the problem is fully understood. Both experiments will again measure the $b$ and $b \bar{b}$ cross sections at higher center-of-mass energy.

To further shed light on this problem, CDF has recently presented a measurement of the charm production cross section. ${ }^{4}$ Using the secondary vertex trigger, CDF has been able to reconstruct very large samples of charm decays. Figure 2 shows a fully reconstructed $D^{+} \rightarrow K^{-} \pi^{+} \pi^{+}$signal using $5.8 \mathrm{pb}^{-1}$ of data from early in the run. In the full data sample available at the time of this Symposium $\left(\mathcal{L} \sim 200 \mathrm{pb}^{-1}\right)$, CDF has a $D^{0}$ sample exceeding 2 million events. As this sample grows, competitive searches for $C P$-violation in the charm sector and $D^{0} / \overline{D^{0}}$ mixing are anticipated.

Since the events are accepted based upon daughter tracks with large impact parameter, it is clear that the sample of reconstructed charm decays contains charm from bottom $(p \bar{p} \rightarrow b \bar{b} X$, with $b \rightarrow$ $c \rightarrow D)$ in addition to prompt charm production $(p \bar{p} \rightarrow c \bar{c} X$, with $c \rightarrow D$.) To extract the charm meson cross section, it is necessary to extract the fraction of $D$ mesons that are coming from prompt charm production. This is done by measuring the impact parameter of the charm meson. If it arises from direct $c \bar{c}$ production, the charm meson will have a small impact parameter pointing back to the point of production, which was the collision vertex. If the charm meson arises from $b$ decay, it will typically not 


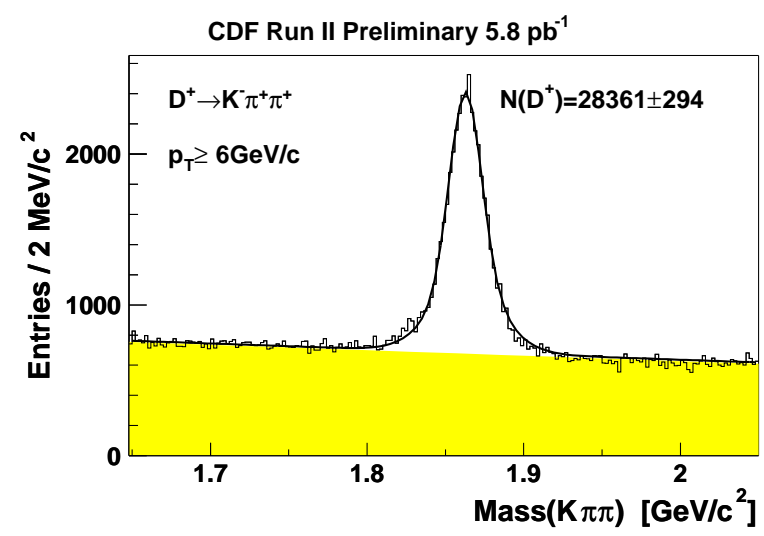

Figure 2. Yield for $D^{+} \rightarrow K^{-} \pi^{+} \pi^{+}$used in the charm cross section analysis. Yields of the size shown in the plot are routinely acquired in a single week of data taking. This sample was acquired using the CDF SVT trigger. About $80 \%$ of the signal in this sample arises from direct charm production.

extrapolate back to the primary vertex.

Using this technique, along with a sample of $K_{S}^{0} \rightarrow \pi^{+} \pi^{-}$decays for calibration, it is determined that $80-90 \%$ (depending upon the mode) of the charm mesons arise from direct charm production. The shorter charm lifetime is more than compensated by the copious charm production in the high energy collisions.

The full analysis includes measurements of the differential cross sections for prompt $D^{0}, D^{+}, D^{*+}$ and $D_{s}^{+}$meson production. The integrated cross section results of this study are summarized in Table 3 . Figure 3 shows the comparison between data and the NLO calculation for the differential $D^{0}$ cross section. ${ }^{5}$ The trend seen in this figure is the same for the other $D$ species. The prediction seems to follow the measured cross section in shape, but the absolute cross section is low compared to the measured results. This difference in magnitude between the measured and predicted charm meson cross section is similar to the difference in data and theory seen in the $B$ meson cross sections.

As an interesting aside, we can also compare the measured $B$ and $D$ cross sections at the Tevatron. ${ }^{3}$ Looking at the charged mesons, the measured cross sections are:

$$
\begin{gathered}
\sigma\left(D^{+}, p_{T} \geq 6 \mathrm{GeV} / c,|y| \leq 1\right)=4.3 \pm 0.7 \mu \mathrm{b} \\
\sigma\left(B^{+}, p_{T} \geq 6 \mathrm{GeV} / c,|y| \leq 1\right)=3.6 \pm 0.6 \mu \mathrm{b} .
\end{gathered}
$$

For this momentum range, the $D^{+}$cross section is only $20 \%$ larger than the $B^{+}$cross section. At very

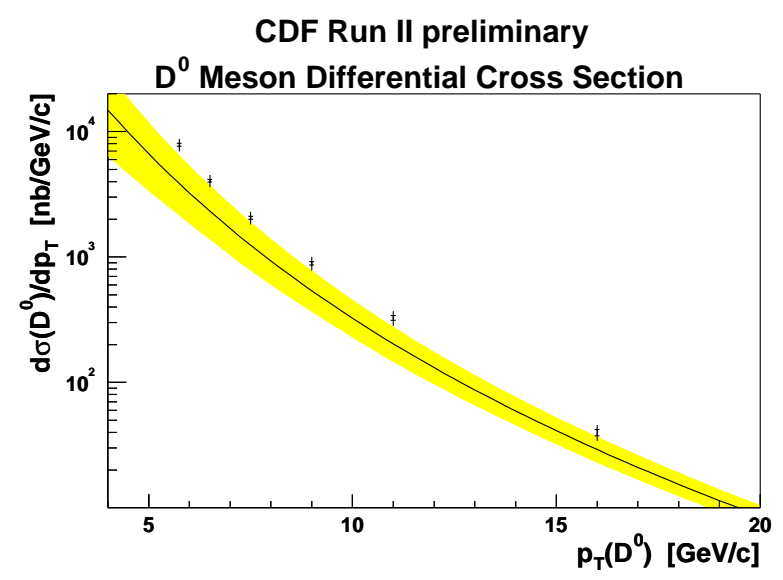

Figure 3. The measured differential cross section for prompt $p \bar{p} \rightarrow D^{0} X$, with $\left|y\left(D^{0}\right)\right|<1$. The $D^{0}$ hadrons arising from $B$ decays have been removed. The NLO calculation is from Cacciari and Nason. ${ }^{5}$ The results for the other charm modes are summarized in Table 3.

Table 3. CDF measurement of the direct charm cross section. The results are for $D$ mesons with $|y|<1$.

\begin{tabular}{ccc} 
Meson & Momentum range & Measured cross section $(\mathrm{pb})$ \\
\hline$D^{0}$ & $p_{T}>5.5 \mathrm{GeV} / c$ & $13.3 \pm 0.2 \pm 1.5$ \\
$D^{*+}$ & $p_{T}>6.0 \mathrm{GeV} / c$ & $5.2 \pm 0.1 \pm 0.8$ \\
$D^{+}$ & $p_{T}>6.0 \mathrm{GeV} / c$ & $4.3 \pm 0.1 \pm 0.7$ \\
$D_{s}^{+}$ & $p_{T}>8.0 \mathrm{GeV} / c$ & $0.75 \pm 0.05 \pm 0.22$ \\
\hline
\end{tabular}

high transverse momentum (corresponding to high $Q^{2}$ ), we would expect that the mass difference between the bottom and charm quarks to be a small effect, yielding similar production cross sections. However these results show that even at lower $p_{T}$, the mass effects are not that significant.

\section{$6 \quad B$ Lifetimes}

In the spectator model for meson decays, where the light quark does not participate in the decay, all $B$ lifetimes are equal, since the lifetime is exclusively determined by the lifetime of the $b$ quark. In reality, non-spectator effects such as interference modify this expectation. The Heavy Quark Expansion (HQE) predicts the lifetime hierarchy for the $B$ hadrons as:

$$
\tau\left(B^{+}\right)>\tau\left(B^{0}\right) \simeq \tau\left(B_{s}\right)>\tau\left(\Lambda_{b}\right)>>\tau\left(B_{c}\right),
$$

where the $B_{c}$ meson is expected to have the shortest lifetime because both the $b$ and the $c$ quarks are able to decay by the weak interaction. 
The lifetimes of the light mesons, $B^{0}$ and $B^{+}$, are measured with a precision that is better than $1 \%$. This impressive level of precision is dominated by the measurements of the Belle and Babar experiments. ${ }^{6}$ The $B_{s}$ and $b$-baryon lifetimes have been measured by the LEP, SLD and CDF Run I experiments. One interesting puzzle that persists from those measurements is that the $\Lambda_{b}$ lifetime is significantly lower than expectation. ${ }^{?}$

To measure the lifetime of a particle, the experiments utilize their precision silicon tracking to measure the flight distance of the hadron before it decays. At the Tevatron, this is done in the plane transverse to the beamline, and the two-dimensional flight distance is denoted as $L_{x y}$. Since the particle is moving at a high velocity in the lab frame, the decay time measured in the laboratory is dilated relative to the proper-decay time, which is the decay time of the particle in its rest frame. To extract the proper decay time, we must correct for the time dilation factor:

$$
c t_{\text {decay }}=\frac{L_{x y}}{(\beta \gamma)_{T}}
$$

where $t_{\text {decay }}$ is the proper decay time in the rest frame of the particle, $c$ is the speed of light, $\beta \gamma=$ $v / c \sqrt{1-v^{2} / c^{2}}$ is the relativistic correction for the time dilation. We write $(\beta \gamma)_{T}=p_{T} / m_{B}$, with $p_{T}$ the transverse momentum of the $B$ hadron and $m_{B}$ the mass of the $B$ hadron. The quantity $c_{\text {decay }}$ is referred to as the proper decay length.

The uncertainty in the measurement of the proper decay length $\left(\sigma_{c t}\right)$ has three terms:

$$
\sigma_{c t}=\left(\frac{m_{B}}{p_{T}}\right) \sigma_{L_{x y}} \otimes c t\left(\frac{\sigma_{p_{T}}}{p_{T}}\right) \otimes\left(\frac{L_{x y}}{p_{T}}\right) \sigma_{m_{B}}
$$

where the $\otimes$ symbol indicates that the terms combine in quadrature. The final term, which is proportional to the uncertainty on the $B$ hadron mass $\left(\sigma_{m_{B}}\right)$ is negligible in all cases. The first term is the uncertainty on the measured decay length. This depends upon the resolution of the detector as well as the topology and momentum of the decay mode. The middle term, proportional to the uncertainty transverse momentum of the $B$ hadron $\left(\sigma_{p_{T}}\right)$ is effectively the uncertainty in the time dilation correction. For fully reconstructed modes where all of the $B$ daughter particles are accounted for, this term provides a negligible contribution to the uncertainty on the

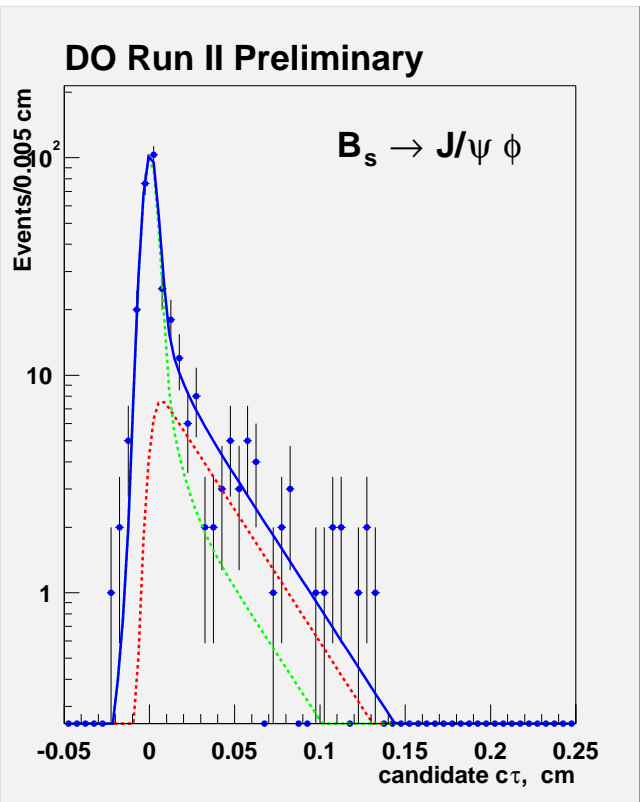

Figure 4. A measurement of the lifetime of the $B_{s}$ meson from the $\mathrm{D} \varnothing$ experiment. This result uses the fully reconstructed $B_{s} \rightarrow J / \psi \phi$ mode, with $\phi \rightarrow K^{+} K^{-}$. The signal yield is $133 \pm$ 17 events in $115 \mathrm{pb}^{-1}$ of data. The solid curve is the combined signal + background fit to the data, while the dashed curves are the data and background components shown separately. The background dominates at zero decay time, but there is also a background contribution coming from heavy flavor.

proper decay time. In the case of partially reconstructed modes, where some fraction of the $B$ daughters are not reconstructed, the uncertainty on the $B$ hadron momentum becomes a significant contributor to the lifetime uncertainty.

Fully reconstructed $J / \psi$ modes, such as $B^{0} \rightarrow$ $J / \psi K^{* 0}$, with $K^{* 0} \rightarrow K^{-} \pi^{+}$have the advantage of having small uncertainty in the $p_{T}$ of the $B$ hadron. The drawback, however, is that the signal yields are small due to the small branching ratio into the color-suppressed $J / \psi$ mode. Figure 4 shows a measurement of the $B_{s}$ lifetime from $\mathrm{D} \varnothing$ in the mode $B_{s} \rightarrow J / \psi \phi$, with $J / \psi \rightarrow \mu^{+} \mu^{-}$and $\phi \rightarrow K^{+} K^{-}$. In fitting for the lifetime, it is necessary to account for backgrounds from prompt sources as well as backgrounds from heavy flavor sources. In the case of the fully reconstructed modes, the lifetime fit can additionally utilize reconstructed mass information to properly weight signal versus background events. In the case of $J / \psi$ modes, the dominant backgrounds come from real $J / \psi$ decays from both prompt $c \bar{c}$ production as well as $B$ decays. 


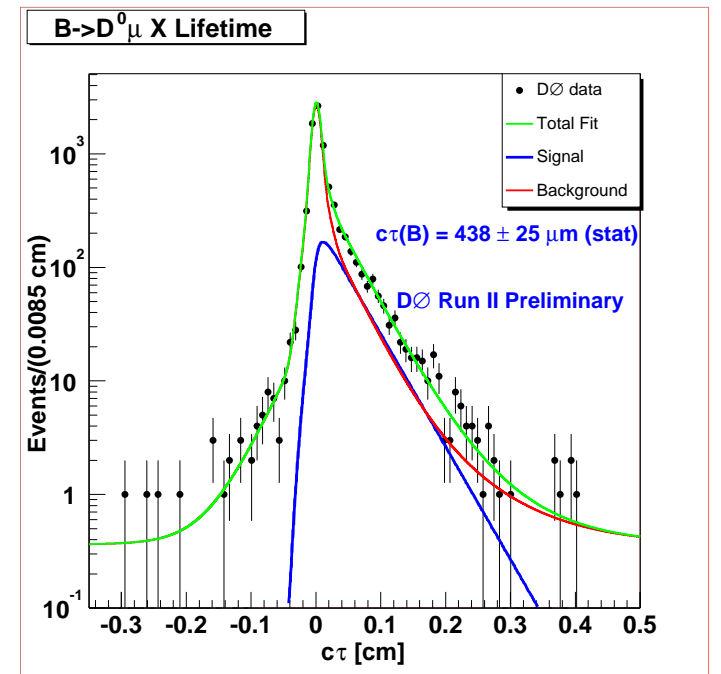

Figure 5. A measurement of the inclusive $B \rightarrow \mu D^{0} X$ lifetime from the $\mathrm{D} \varnothing$ experiment. This inclusive lifetime has contributions from all $B$ modes, although the sample is dominantly $B^{+}$and $B^{0}$ decays.

The statistical uncertainties on the D $\varnothing$ and CDF lifetime measurements are not yet competitive with the current world average for the $B_{s}$ and $\Lambda_{b}$ lifetimes. With larger data samples over the next 1-2 years, new results from the Tevatron will surpass the current level of precision.

Alternatively, semileptonic decays, such as $B_{s}^{0} \rightarrow$ $D_{s}^{-} \ell \nu_{\ell}$, with $D^{-} \rightarrow \phi \pi^{-}$provide larger signal yields but suffer from uncertainty in the $B$ hadron $p_{T}$ due to the unreconstructed neutrino. With large data samples, the semileptonic modes will begin to become systematics limited due to the partial reconstruction, while the statistics limited fully reconstructed modes will continue to provide improved sensitivity.

Figure 5 shows a measurement of the inclusive $B$ lifetime in the $\mu D^{0}$ mode. Since this is a partial reconstruction, backgrounds can be more challenging than they are in the fully reconstructed mode. One technique to suppress backgrounds is to demand the proper charge correlation between the muon and the $D^{0}$ meson. The charge of the charm quark is carried through the $D^{0}$ decay $\left(D^{0} \rightarrow K^{-} \pi^{+}\right.$and $\overline{D^{0}} \rightarrow K^{+} \pi^{-}$) so the correlation that the charge of the muon be the same as the charge of the kaon can be enforced to reduce backgrounds. This works even without particle identification, because if the $K$ and $\pi$ masses are assigned incorrectly they typically do not reconstruct a $D^{0}$ mass.

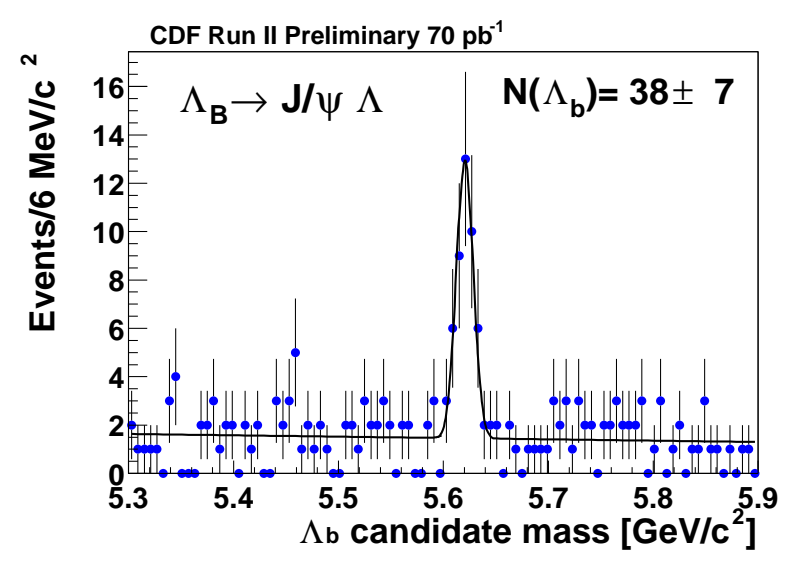

Figure 6. A measurement of the mass of the $\Lambda_{b}$ baryon from the CDF experiment. The fully reconstructed $\Lambda_{b} \rightarrow J / \psi \Lambda$, with $J / \psi \rightarrow \mu^{+} \mu^{-}$and $\Lambda \rightarrow p \pi^{-}$is shown here. The mass scale is calibrated with very high precision using $J / \psi, \psi(2 S)$ and $\Upsilon \rightarrow \mu^{+} \mu^{-}$decays.

One interesting background to the semileptonic analysis is $c \bar{c}$ production where one charm hadron decays semileptonically and the other fragments into a $D^{0}$. This background is only a problem if the charm pair is produced at a very small opening angle, which is exactly what occurs when a hard gluon splits into a $c \bar{c}$ pair. This "gluon splitting" contribution produces the "right-sign" charge correlation between the $D$ and muon. Even though the $D^{0}$ extrapolates back to the primary vertex in this case, the "fake" $\mu D^{0}$ vertex can look like it arose from a long-lived state. Further studies of $c \bar{c}$ production correlations are needed to fully understand this background source.

\section{B Hadron Masses}

CDF has performed precision measurements of the masses of $B$ hadrons using fully reconstructed $B \rightarrow$ $J / \psi X$ modes. High statistics $J / \psi \rightarrow \mu^{+} \mu^{-}$and $\psi(2 S) \rightarrow J / \psi \pi^{+} \pi^{-}$are used to calibrate tracking momentum scale and material in the tracking volume. The results are tabulated in Table 4. Figure 6 shows the measurement of the $\Lambda_{b}$ baryon mass. Even with relatively small statistics (less than 100 events in $B_{s}$ and $\Lambda_{b}$ modes) these new results are the world's best measurements of these masses. 
Table 4. CDF results on masses of $B$ hadrons. These results come from fully reconstructed $J / \psi$ modes. The first error is statistical, the second systematic.

\begin{tabular}{clc}
$B$ hadron & Decay mode & Measured mass $\left(\mathrm{MeV} / c^{2}\right)$ \\
\hline$B^{+}$ & $J / \psi K^{+}$ & $5279.32 \pm 0.68 \pm 0.94$ \\
$B^{0}$ & $J / \psi K^{* 0}$ & $5280.30 \pm 0.92 \pm 0.96$ \\
$B_{s}^{0}$ & $J / \psi \phi$ & $5365.50 \pm 1.29 \pm 0.94$ \\
$\Lambda_{b}$ & $J / \psi \Lambda$ & $5620.4 \pm 1.6 \pm 1.2$ \\
\hline
\end{tabular}

\section{Hadronic Branching Ratios}

\subsection{Two-body Charmless B Decays}

With the new SVT trigger, CDF has begun to measure $B$ decays with non-leptonic final states. One set of modes of particular interest are the charmless two-body modes. Requiring the final state to consist of two charged hadrons, the following modes can be accessed at the Tevatron:

- $B^{0} \rightarrow \pi^{+} \pi^{-}, B R \sim 5 \times 10^{-6}$

- $B^{0} \rightarrow K^{+} \pi^{-}, B R \sim 2 \times 10^{-5}$

- $B_{s} \rightarrow K^{+} K^{-}, B R \sim 1 \times 10^{-5}$

- $B_{s} \rightarrow K^{-} \pi^{+}, B R \sim 2 \times 10^{-6}$.

The $B^{0}$ states are accessible at the $e^{+} e^{-}$facilities, but the $B_{s}$ modes are exclusive to the Tevatron.

The measurement presented here is the first observation of the decay $B_{s} \rightarrow K^{+} K^{-}$. As more data is accumulated, the longer term goal from these modes is to search for direct $C P$-violation as well as measure the $C K M$ angle $\gamma=\operatorname{Arg}\left(V_{u b}^{*}\right){ }^{8}$

Figure 7 shows the reconstructed signal where all tracks are assumed to have the mass of the pion. A clear peak is seen, and the width of the peak is significantly larger $\left(41 \mathrm{MeV} / c^{2}\right)$ than the intrinsic resolution of the detector. This additional width is due to the $K^{+} \pi^{-}$and $K^{+} K^{-}$final states from $B^{0}$ and $B_{s}$ decays. To extract the relative contributions, kinematic information and $d E / d x$ particle identification is used. ${ }^{c}$ The particle identification is calibrated from a large sample of $D^{*+} \rightarrow D^{0} \pi^{+}$decays, with $D^{0} \rightarrow K^{-} \pi^{+}$. The charge of the pion from the $D^{*}$ uniquely identifies the kaon and pion, providing an excellent calibration sample for the $d E / d x$ system. Although the $K-\pi$ separation is $1.3 \sigma$, this is sufficient to extract the two-body $B$ decay contributions.

\footnotetext{
${ }^{c} \mathrm{CDF}$ has a time-of-flight system for particle identification with very good $\pi-K$ separation for track $p_{T}<1.6 \mathrm{GeV} / c$. The tracks from these two-body decay modes have $p_{T}>2 \mathrm{GeV} / c$ and therefore the time-of-flight system does not provide additional particle identification information for this analysis.
}

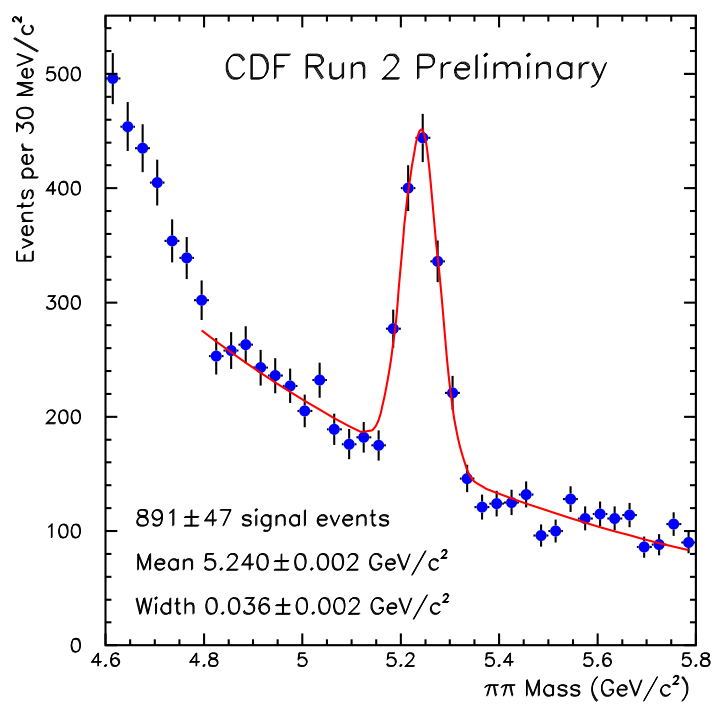

Figure 7. The reconstructed two-body $B \rightarrow h^{+} h^{-\prime}\left(h, h^{\prime}=K\right.$ or $\pi$ ) sample from CDF. For this plot, all particles are reconstructed as pions. The peak consists of contributions from the four modes listed in the text. These pieces are extracted using kinematic and particle identification information. The luminosity used for the figure shown here is $\mathcal{L}=190 \mathrm{pb}^{-1}$. The results presented in the text are for a subset of this data.

The results from $\mathcal{L}=65 \mathrm{pb}^{-1}$ are shown in Table 5 . The $B^{0}$ modes have been measured by CLEO, Babar and Belle. This is the first observation of the $B_{s}^{0} \rightarrow K^{+} K^{-}$decay mode. Turning the yields into a ratio of branching ratios, the result is:

$$
\frac{B R\left(B_{s}^{0} \rightarrow K^{+} K^{-}\right)}{B R\left(B_{d}^{0} \rightarrow K^{+} \pi^{-}\right)}=2.71 \pm 1.15
$$

where the error is the combined statistical and systematic uncertainty. To calculate this ratio, information about the relative production rates of $B_{s}^{0}$ and $B^{0}$ mesons must be included. The uncertainty includes the uncertainty on the relative production fractions.

\section{2 $\Lambda_{b} \rightarrow \Lambda_{c} \pi^{-}$Branching Ratio}

Using the SVT trigger, CDF has begun to measure $b$-baryon states. Figure 8 shows a clean signal of the decay $\Lambda_{b} \rightarrow \Lambda_{c} \pi^{-}$, with $\Lambda_{c} \rightarrow p K^{-} \pi^{+}$. The reconstructed invariant mass plot has a very interesting structure, with almost no background above the peak and a background that rises steeply in going to lower mass. This structure is somewhat unique to baryon modes, which are the most massive weakly 
Table 5. CDF results on two-body charmless $B$ decays. The yields reported in this table are extracted by fitting the decay information for the events, including kinematic and particle identification information. Yields shown here are for $\mathcal{L} \sim$ $65 \mathrm{pb}^{-1}$.

\begin{tabular}{lr} 
Mode & \multicolumn{1}{c}{ Fitted yield (events) } \\
\hline$B^{0} \rightarrow K^{-} \pi^{+}$ & $148 \pm 17($ stat.) $\pm 17($ syst. $)$ \\
$B^{0} \rightarrow \pi^{+} \pi^{-}$ & $39 \pm 14($ stat. $) \pm 17$ (syst.) \\
$B_{s}^{0} \rightarrow K^{+} K^{-}$ & $90 \pm 17($ stat. $) \pm 17($ syst. $)$ \\
$B_{s}^{0} \rightarrow K^{-} \pi^{+}$ & $3 \pm 11($ stat. $) \pm 17$ (syst.) \\
\hline
\end{tabular}

decaying $B$ hadron states. Because the SVT trigger specifically selects long-lived states, most of the backgrounds are coming from other heavy flavor $(b$ and $c$ ) decays. Since there are no weakly decaying $B$ hadrons more massive than the $\Lambda_{b}$, there is very little background above the peak. On the other hand, going to masses below the peak, lighter $B$ mesons begin to contribute. The background in this mode is growing at lower masses because there is more phase space for $B^{+}, B^{0}$, and $B_{s}^{0}$ to contribute.

To extract the number of signal events, $b \bar{b}$ Monte Carlo templates are used to account for the reflections seen in the signal window. The shapes of these templates are fixed by the simulation, but their normalization is allowed to float. The number of fitted signal events in this analysis is $96 \pm$ $13(\text { stat. })_{-7}^{+6}$ (syst.). The primary result from this analysis is a measurement of the $\Lambda_{b} \rightarrow \Lambda_{c} \pi^{-}$branching ratio relative to the $B^{0} \rightarrow D^{-} \pi^{+}$mode. We can take that ratio, along with PDG $2002^{9}$ values for measured branching ratios and production fractions, and extract the branching ratio

$$
B R\left(\Lambda_{b} \rightarrow \Lambda_{c} \pi^{-}\right)=(6.5 \pm 1.1 \pm 0.9 \pm 2.3) \times 10^{-3},
$$

where the errors listed are statistical, systematic and the final uncertainty is arising from the uncertainty in the $B^{0} \rightarrow D^{-} \pi^{+}$branching ratio.

\subsection{Observation of the $X(3872)$ State}

At this Lepton-Photon Symposium, the Belle collaboration announced the observation of a neutral state decaying into $J / \psi \pi^{+} \pi^{-}$with a mass of $3872 \mathrm{MeV} .^{10}$ This state may be the $1^{3} D_{2} c \bar{c}$ bound state, although the observed mass is higher than expected for that state. It has also been hypothesized that this is a loosely bound $D \bar{D}^{*}$ bound state, since the mass is right at the $D \bar{D}^{*}$ threshold.

Belle observes this state in $B$ decays. Their ob-

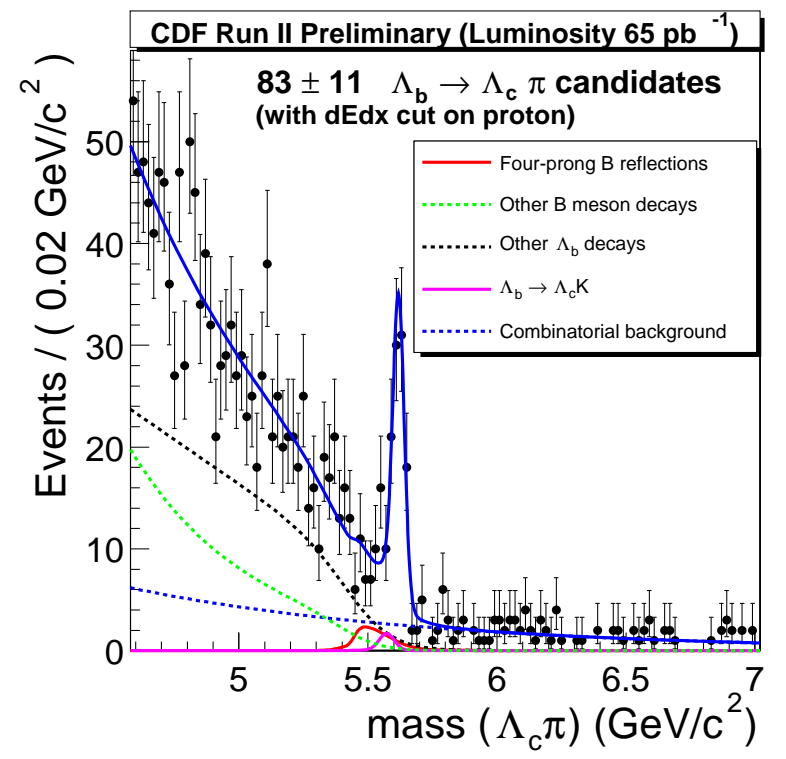

Figure 8. The CDF fully reconstructed $\Lambda_{b} \rightarrow \Lambda_{c} \pi$, with $\Lambda_{c} \rightarrow$ $p K^{-} \pi^{+}$. The features in the sidebands are discussed in the text. For this plot, an additional particle identification cut on the proton was made that was not used in the extraction of the branching ratio.

servation further indicates that the state is narrow, and favors large $\pi^{+} \pi^{-}$mass in the decay.

CDF has searched for this state using a sample of $\mathcal{L} \sim 220 \mathrm{pb}^{-1}$ and sees a clear signal with a mass of

$$
3871.4 \pm 0.7 \text { (stat. }) \pm 0.4(\text { syst. }) \mathrm{MeV} / c^{2} .
$$

Figure 9 shows the CDF $J / \psi \pi^{+} \pi^{-}$mass spectrum. This plot originates from a parent sample of approximately 2.2 million $J / \psi \rightarrow \mu^{+} \mu^{-}$decays. The large peak at $3.685 \mathrm{MeV} / c^{2}$ is the $\psi(2 S) .{ }^{11}$

The plot shows events where the dipion mass was required to be greater than $500 \mathrm{MeV} / c^{2}$. The significance of the signal without this cut is greater than $10 \sigma$. CDF also reports that the state is narrow. The observed width of $4.3 \mathrm{MeV} / c^{2}$ is consistent with detector resolution. Further studies are underway to investigate the $\pi^{+} \pi^{-}$mass distribution as well as to determine whether or not the CDF signal is coming from a prompt source or though $B$ decays. Since all angular momentum states are accessible in high energy $p \bar{p}$ collisions, it is possible that this state is directly produced at the Tevatron, while it cannot be directly produced in $e^{+} e^{-}$collisions. 


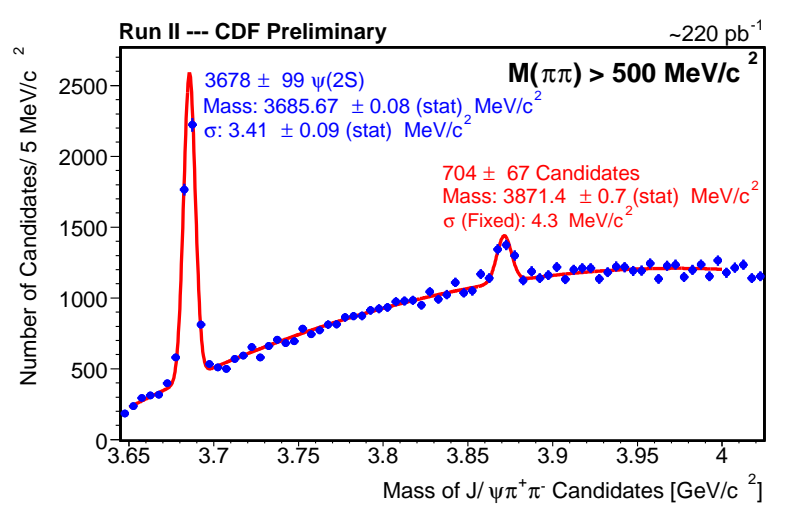

Figure 9. The CDF observation of a neutral state of mass $3871 \mathrm{MeV} / c^{2}$ decaying to $J / \psi \pi^{+} \pi^{-}$This is a confirmation of the state first reported by the Belle collaboration at this conference. The distribution shown here includes a requirement that the dipion mass have $M(\pi \pi)>500 \mathrm{MeV} / c^{2}$. The signal is clear and significant without any cut on the dipion mass.

\section{Mixing}

In the $K^{0}$ and $B^{0}$ systems, particle-antiparticle mixing has been observed and measured with great precision. This mixing is understood to occur because the weak interaction eigenstates are not the same as the strong interaction (or flavor) eigenstates. The weak eigenstates are then linear combinations of the flavor eigenstates, giving rise to an oscillation frequency that is proportional to the mass difference between the heavy and light states.

Recently, the Babar and Belle experiments have made very precise measurements of $B^{0} \overline{B^{0}}$ mixing and have significantly improved the world average. The mixing parameter is typically reported in terms of the heavy/light mass difference. For the $B^{0}$ system, the world average is: ${ }^{7}$

$$
\Delta m_{d}=0.502 \pm 0.006 \mathrm{ps}^{-1} .
$$

From this, we see that a beam of pure $B^{0}$ mesons would result in a beam of pure $\overline{B^{0}}$ mesons in time $\Delta m_{d} t_{m i x}=\pi$, which indicates that $t_{\text {mix }} \sim 4.1$ lifetimes, indicating that the oscillation is rather slow.

Mixing proceeds via a second-order weak transition as shown in Fig. 10. The box diagram includes the $V_{t d}$ matrix element for $B^{0} / \overline{B^{0}}$ mixing, which is replaced by $V_{t s}$ for $B_{s}^{0} / \overline{B_{s}^{0}}$ mixing. Experimentally, we know that $V_{t s}$ is larger than $V_{t d}$ :

$$
\operatorname{Re}\left(V_{t s}\right) \simeq 0.040>\operatorname{Re}\left(V_{t d}\right) \simeq 0.007,
$$

so we expect the $B_{s}^{0}$ system to oscillate with a much



Figure 10. The box diagrams for the $B_{s}^{0} \rightarrow \overline{B_{s}^{0}}$ transition. All up-type quarks $(u, c, t)$ are included in the box, but since these terms are proportional to the mass of the quark in the box, the top quark dominates. Therefore the $V_{t s}$ element is relevant for $B_{s}$ mixing and the $V_{t d}$ element is relevant for $B^{0}$ mixing.

higher frequency than the $B^{0}$ system. Indeed this is the case, the $B_{s}^{0}$ system oscillates so quickly that the oscillations have not yet been resolved. The current combined world limit is: ${ }^{7}$

$$
\Delta m_{s}>14.4 \mathrm{ps}^{-1} @ 95 \% \mathrm{CL},
$$

which means a beam of $B_{s}^{0}$ mesons would fully become a beam of $\overline{B_{s}^{0}}$ mesons in less than $1 / 7^{\text {th }}$ of one lifetime! For the next several years, the Tevatron will be the exclusive laboratory for $B_{s}$ meson studies, including the search for $B_{s}$ mixing.

\subsection{Measuring Mixing}

To measure $B_{s}$ mixing, four ingredients are needed.

- Flavor at the time of production. It is necessary to know whether the meson was produced as a $B_{s}^{0}$ or a $\overline{B_{s}^{0}}$.

- Flavor at the time of decay. It is also necessary to know whether the meson was a $B_{s}^{0}$ or $\overline{B_{s}^{0}}$ when it decayed. This, combined with the flavor at time of production, tells us whether the $B_{s}$ had decayed as mixed or unmixed. ${ }^{d}$

\footnotetext{
"A meson that "decayed as unmixed" could have mixed and mixed back, undergoing one or more complete cycles. This necessarily comes out of the time-dependent analysis.
} 
- Proper decay time. It is necessary to know the proper decay time for the $B_{s}$, since we are attempting to measure the probability to mix as a function of decay time. The $B_{s}$ system mixes too quickly to resolve using timeintegrated techniques.

- Large $B_{s}$ samples. We must map out the probability to mix as a function of decay time for at least part of the decay time spectrum. Because each the previous three items have shortcomings requiring more statistics, this analysis requires large samples of $B_{s}$ decays.

In the following subsections, we will discuss each of these pieces necessary to measure $B_{s}$ mixing.

\subsection{Flavor Tagging}

The first two items in our list of requirements have to do with determining the flavor of the $B_{s}$ meson at the time of production and at the time of decay, referred to as initial-state and final-state flavor tagging.

For initial-state flavor tagging, we infer the flavor of the $B_{s}$ meson at the time of production from other information in the event. Here we can take advantage of what we know about $b \bar{b}$ production. By measuring the flavor of the other $B$ hadron in the event, we can infer the flavor of the $B_{s}$ at the time of production. This technique is imprecise, and also suffers from the fact that quite often $(\sim 75 \%)$ the other $B$ hadron is outside the acceptance of the detector.

Another technique is to look at fragmentation tracks near the $B_{s}$ meson. For a $\bar{b}$ quark to become a $B_{s}^{0}$ meson, it must grab an $s$ quark from the vacuum. When the $s$ is popped from the vacuum, an $\bar{s}$ is popped with it, which could potentially turn into a $K^{+}$meson. We can then use the charge of the kaon to infer the flavor of the $B$ hadron. Again, this is an inexact technique, since other fragmentation tracks can confuse this correlation and also the charge information could be lost into neutral particles, like $K_{S}^{0}$. Figure 11 shows an example of same-side tagging. In this case, a fully reconstructed $B^{+} \rightarrow J / \psi K^{+}$ sample is used, where the flavor of the $B$ hadron is known. The plot then shows the mass difference between the $\pi-B^{+}$and the $B^{+}$. A clear opposite-sign excess is seen over the entire range of the plot, which is attributed to the fragmentation correlation. In addition, a clear peak is seen near $0.4 \mathrm{GeV} / c^{2}$ which is attributed to the $B^{* *}$ state.

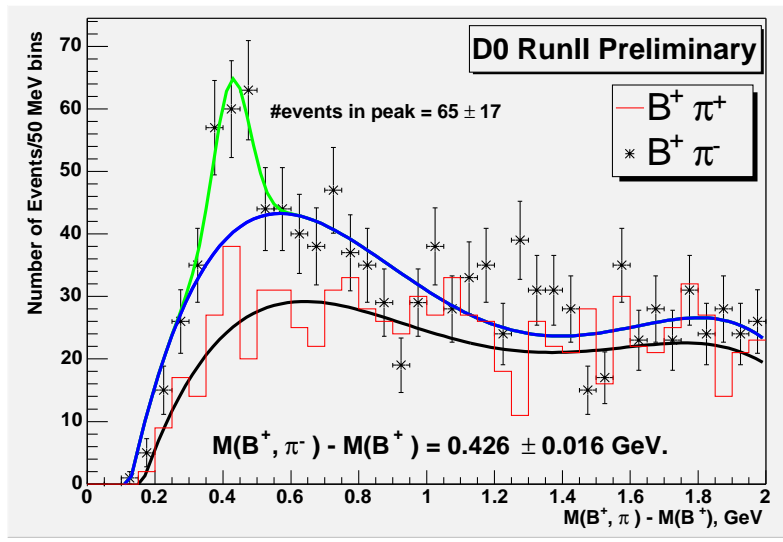

Figure 11. DØ same-side tagging plot.

The efficacy of the initial-state flavor tagging is classified by the tagging power: $\epsilon D^{2}$, where $\epsilon$ is the fraction of times the algorithm was able to arrive at a tagging decision and $D$ is the dilution, which is a measure of the probability that the tag is correct. The dilution is written as: $D=\left(N_{R}-\right.$ $\left.N_{W}\right) /\left(N_{R}+N_{W}\right)$, where $N_{R}\left(N_{W}\right)$ are the number of right (wrong) tags. The dilution is related to the mistag fraction, $w$ as $D=1-2 w$. Tagging power is proportional to $D^{2}$ because incorrectly measuring the sign removes the event from the "correct" charge bin and puts the event into the "wrong" charge bin.

For Babar and Belle, $\epsilon D^{2} \simeq 27 \%$, whereas for the Tevatron $\epsilon D^{2} \simeq 5 \%$. The large difference arises because of the nature and cleanliness of the $e^{+} e^{-}$ environment. As an example, if an experiment has 1000 signal events with $\epsilon D^{2}=5 \%$, then the statistical power of that sample is equivalent to a sample of 50 events where the tag is known absolutely.

For final-state flavor tagging, there are three primary classes of $B_{s}$ decays:

- $B_{s}^{0} \rightarrow D_{s}^{-} \pi^{+}$, with $D_{s}^{-} \rightarrow \phi \pi^{-}$,

- $B_{s}^{0} \rightarrow D_{s}^{-} \ell^{+} \nu_{\ell}$, with $D_{s}^{-} \rightarrow \phi \pi^{-}$,

- $B_{s}^{0} \rightarrow J / \psi \phi$, with $J / \psi \rightarrow \mu^{+} \mu^{-}$.

In the first two cases, the flavor of the $B_{s}$ is immediately evident from the charge of the decay products, which are referred to as a "self-tagging" final states. In the third case, there is no way know whether the meson decayed as a $B_{s}^{0}$ or $\overline{B_{s}^{0}}$, so charge-symmetric modes are of no use for the mixing analysis. ${ }^{e}$

\footnotetext{
${ }^{e}$ The charge-neutral $B_{s}$ modes are important for other analyses, such as the search for a lifetime difference $\Delta \Gamma_{s}$ in $C P$-even and $C P$-odd decays.
} 


\subsection{Proper Decay Time Resolution}

We know the $B_{s}$ oscillates very quickly, therefore we need proper time resolution that is smaller than the oscillation frequency. As discussed in detail in Sec. 6, the two primary components contributing to the proper time resolution are vertex $\left(L_{x y}\right)$ resolution and the time dilation correction $\left((\beta \gamma)_{T}=p_{T} / m_{B}\right)$.

For the semileptonic samples, the time dilation correction factor limits the proper time resolution. For fully reconstructed samples (no missing neutrino) the time dilation correction is a negligible effect and only the $L_{x y}$ resolution contributes to the uncertainty on the proper decay time measurement.

If the true value of $\Delta m_{s}$ is close to the current limit $\Delta m_{s} \sim 14-18 \mathrm{ps}^{-1}$, then both fully reconstructed and semileptonic samples will contribute to the measurement of $\Delta m_{s}$. However, if the true value of $\Delta m_{s}$ is $20 \mathrm{ps}^{-1}$ or higher, then the proper time resolution becomes the limiting factor in resolving the oscillations. At $\Delta m_{s}$ values this high, only the fully reconstructed samples are useful, and in fact the vertex resolution becomes the limiting factor.

\subsection{Yields}

As stated previously, the statistics of the semileptonic samples are higher than those of the fully reconstructed modes. For lower values of $\Delta m_{s}$ the larger semileptonic event yields somewhat offset the poorer proper time resolution. As a consequence, both CDF and $\mathrm{D} \varnothing$ are continuing to acquire semileptonic samples, both for flavor tagging calibration and for the $B_{s}$ mixing search. A semi-muonic sample enriched in $B_{s}$ decays is shown in Fig. 12, demonstrating that large semileptonic samples are being acquired.

The fully reconstructed states offer fewer signal events, but the improved proper time resolution compensates for this at values of $\Delta m_{s}$ that we are considering. CDF has accumulated a sample of fully reconstructed $B_{s}$ decays as shown in Figure 13. The plot on the left is the signal, a clear $B_{s}$ peak can be seen. The broad peak below the $B_{s}$ is the $B_{s} \rightarrow D_{s}^{*} \pi$, where the photon from the $D_{s}^{*}$ decay is not reconstructed. The plot on the right shows the expected contributions from a $b \bar{b}$ Monte Carlo. In the data, the signal and sidebands are fit using the shapes from the Monte Carlo letting the normalizations float. The Monte Carlo clearly provides a very good description of the signal and heavy flavor backgrounds. This

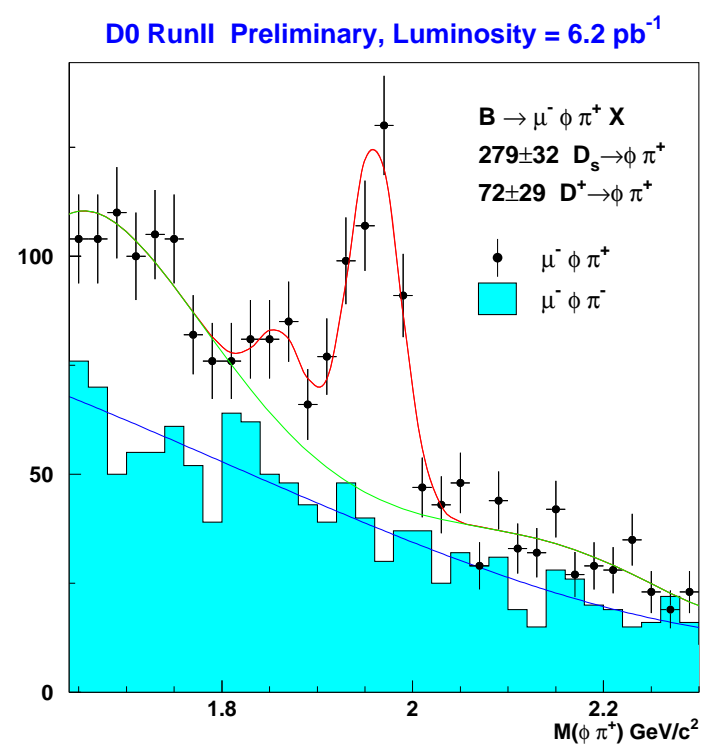

Figure 12. Semileptonic $B_{s}^{0}$ decays can be partially reconstructed in the mode $B_{s}^{0} \rightarrow \ell^{+} D_{s}^{-} X$, with $D_{s}^{-} \rightarrow \phi \pi^{-}$and $\phi \rightarrow K^{+} K^{-}$. The distribution shown here is a sample of $\mu \phi \pi$ events from $\mathrm{D} \varnothing$. The two peaks correspond to the $D^{+}$and $D_{s}^{+}$states, which can both decay to $\phi \pi^{+}$. This mode will provide a large sample of semileptonic $B_{s}^{0}$ decays to search for $B_{s}^{0} / \overline{B_{s}^{0}}$ mixing.

is possible because the SVT trigger provides very pure heavy flavor samples. Even though the hadron collider environment is challenging, clean samples of heavy flavor decays can be isolated.

\section{5 $B_{s}$ Mixing Status and Prospects}

Both experiments have now commissioned the detectors and accumulated the first portion of Tevtron Run 2 data. If we take the current performance of the trigger, reconstruction and flavor tagging, it appears that with a sample of $\mathcal{L} \sim 500 \mathrm{pb}^{-1}$, the $B_{s}$ sensitivity will be comparable to the current combined world limit. To observe or exclude a value of $\Delta m_{s}>15 \mathrm{ps}^{-1}$ will require additional data and improvements along with further progress on triggering, reconstruction and flavor tagging.

With modest improvements to the current running configuration, we estimate that it will take 2$3 \mathrm{fb}^{-1}$ of integrated luminosity to "cover" the region of $\Delta m_{s}$ that is currently preferred by indirect fits. ${ }^{12}$ It is important to recall that the current combined world limit consists of contributions from 13 different measurements, and is the culmination of the LEP, 

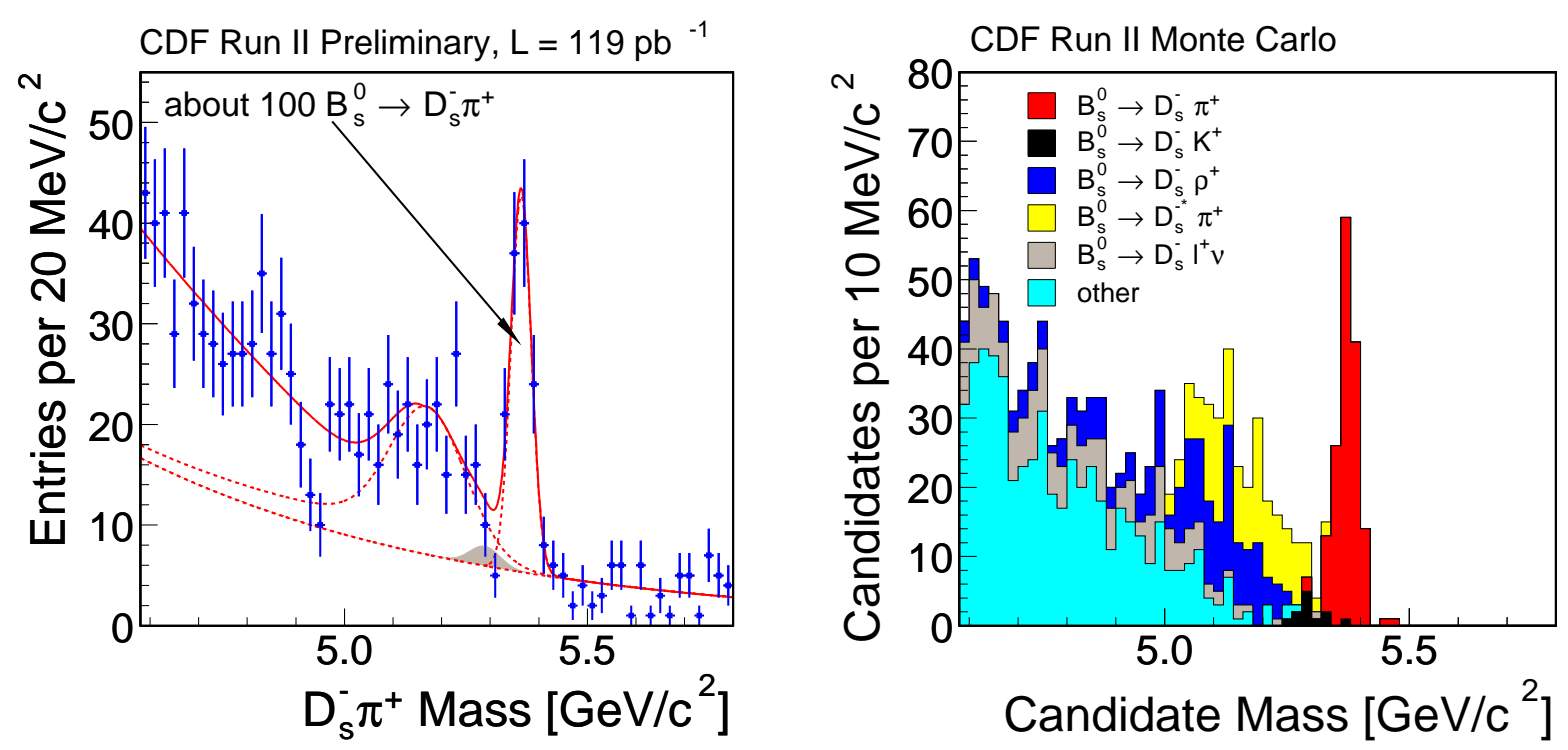

Figure 13. Fully reconstructed $B_{s}^{0}$ decays from CDF using the SVT trigger. The decay chain is $B_{s}^{0} \rightarrow D_{s}^{-} \pi^{+}$, with $D_{s}^{-} \rightarrow \phi \pi^{-}$ and $\phi \rightarrow K^{+} K^{-}$. For the mixing analysis, the proper time resolution will be better in this mode, because the error on the time-dilation factor is negligible. The challenge is the limited statistics. CDF is currently trying to reconstruct other modes to supplement this sample.

SLD and CDF Run I programs. Thanks to additional luminosity and upgraded detectors, it will be possible to extend this search for $B_{s}$ mixing, however this is a very challenging measurement that will take time, effort and a significant data sample.

\section{Summary}

The results shown in this summary provide a snapshot of the heavy flavor results coming from the Tevatron. The period of commissioning is now complete, and the experiments are slightly more than one year into a multi-year run that will continue to accumulate large data samples. The upgraded detectors are performing well, and many of the upgrades specific to $B$ physics are beginning to pay off. Over the next several years, CDF and D $\varnothing$ will make significant contributions in our understanding of production and decay of heavy flavors.

\section{Acknowledgments}

I would like to thank the Lepton-Photon 2003 organizers for the opportunity to speak at this excellent conference. I would also like to thank and acknowledge the collaborators of the Babar, Belle, CDF and
DØ experiments. This work is supported by the U.S. Department of Energy Grant DE-FG02-91ER40677.

\section{References}

1. D $\varnothing$ Collaboration, FERMILAB-Pub-96/357-E, 1996.

2. CDF Collaboration, FERMILAB-Pub-96/390-E, 1996; Fermilab-Proposal-909, 1998.

3. CDF Collaboration (D. Acosta et al.), Phys. Rev. D65, 052005, (2002).

4. CDF Collaboration (D. Acosta et al.), FERMILABPUB-03-217-E, hep-ex/0307080, July 2003.

5. M. Cacciari and P. Nason, hep-ph/0306212, JHEP 0309:006 (2003), June 2003.

6. BABAR Collaboration (B. Aubert et al.), Phys. Rev. Lett. 87, 201803 (2001); BELLE Collaboration (K. Abe et al.), Phys. Rev. Lett. 88, 171801 (2002).

7. Heavy Flavor Averaging Group, http://www.slac. stanford.edu/xorg/hfag/index.html.

8. R. Fleischer, Phys. Lett. B459, 306-320 (1999).

9. Particle Data Group, http://pdg.lbl.gov/.

10. BELLE Collaboration (K. Abe et al.), hepex/0308029, August 2003; S.-K. Choi et al., hepex/0309032, September 2003, submitted to PRL.

11. CDF Collaboration, http://www-cdf.fnal.gov/ physics/new/bottom/030224.blessed-x3872/

12. M. Ciuchini, et al., hep-ph/0307195, April 2003. 


\section{DISCUSSION}

Jonathan L. Rosner (University of Chicago): What are the prospects for seeing fully reconstructed $B_{c}$ mesons, e.g. in $J / \psi \pi^{ \pm}$?

Kevin Pitts: In Run I with $\mathcal{L} \simeq 100 \mathrm{pb}^{-1}$, the $B_{c}$ was observed through the decay $B_{c} \rightarrow J / \psi \ell \nu_{\ell}$, with $\ell=e, \mu$ and $J / \psi \rightarrow \mu^{+} \mu^{-}$, providing a tri-lepton final state. In addition, there was a hint of a signal in $B_{c} \rightarrow J / \psi \pi^{ \pm}$. In both of these modes, the $b$ quark decays to charm, providing the $J / \psi$ in the final state. In Run II, with $\mathcal{L} \sim 220 \mathrm{pb}^{-1}$ already on tape the prospects are quite good both for semileptonic decay and for fully reconstructed decays. In addition, with the enough statistics in the hadronic trigger, it is likely that $\mathrm{CDF}$ can reconstruct the $B_{c}$ in modes where the charm quark decays, such as $B_{c}^{+} \rightarrow B_{s}^{0} \pi^{+}$. This mode might be especially interesting as a new tagging mode for $B_{s}^{0}$ mixing.

Vivek Sharma (University of California at San Diego): What is the timescale for a $10 \%$ measurement of $\Lambda_{b}$ lifetime? In particular can you use the various fully reconstructed hadronic $\Lambda_{b}$ modes given the reflection and impact parameter bias?

Kevin Pitts: Performing a simple average of the $\mathrm{CDF}$ and $\mathrm{D} \varnothing$ measurements of the $\Lambda_{b}$ lifetime in $\Lambda_{b} \rightarrow J / \psi \Lambda$, the result is $\tau_{\Lambda_{b}}=1.13 \pm 0.18 \mathrm{ps}$, which is a $16 \%$ measurement on the $\Lambda_{b}$ lifetime. These results do not yet use the entire Run II data samples available. Assuming all errors scale like $1 / \sqrt{N}$, the combined result from the two experiments using the full $\mathcal{L} \sim 220 \mathrm{pb}^{-1}$ on tape as of this conference, the combined result would be a $10 \%$ measurement on the $\Lambda_{b}$ lifetime. With more data coming in, it seems safe to expect that each experiment will have a measurement in the neighborhood of $10 \%$ by the summer of 2004 .

As for the hadronic modes, significant progress has been made in understanding both the reflections and the impact parameter bias. The understanding of the reflections has been shown in the context of the $\Lambda_{b} \rightarrow \Lambda_{c} \pi$ branching ratio measurement. Understanding the lifetime bias coming from the SVT trigger is a necessity for $B_{s}$ mixing, and all lifetime measurements will benefit from ongoing progress on that front.

Vera Luth (SLAC): For charm or $B$ decays you can normalize your measured $B R$ to other measurements. How are you planning to obtain absolute $B R$ and production rates for $B_{s}, \Lambda_{b}$, etc?

Kevin Pitts: We can still normalize our branching ratios to other modes, but you point out an additional complication coming about due to the lack of precision in our measurements of the relative production fractions. For example, in the CDF measurement of the branching ratio for $\Lambda_{b} \rightarrow \Lambda_{c} \pi$, the number of signal events in two modes is measured: $N\left(\Lambda_{b} \rightarrow \Lambda_{c} \pi\right)$ and $N\left(B^{0} \rightarrow D^{-} \pi^{+}\right)$. The efficiencies and acceptances are calculated, and the PDG values for the $B R\left(D^{-} \rightarrow K^{-} \pi^{+} \pi^{+}\right)$and $B R\left(\Lambda_{c} \rightarrow\right.$ $p K^{-} \pi^{+}$are used. Combining this, the measured ratio of corrected yields gives:

$$
\frac{f_{\text {baryon }} \times B R\left(\Lambda_{b} \rightarrow \Lambda_{c} \pi^{-}\right)}{f_{d} \times B R\left(B^{0} \rightarrow D^{-} \pi^{+}\right)}
$$

where $f_{\text {baryon }}\left(f_{d}\right)$ are the fraction of produced $\Lambda_{b}\left(B^{0}\right)$ hadrons in $p \bar{p}$ collisions.

The extra piece that comes about in taking this ratio is the ratio of production fractions. These are not known very well, and it is important to improve upon our knowledge of the production fractions. This is easier said than done. One way to improve upon our knowledge of $f_{s} / f_{d}$ is to measure $\bar{\chi}$, the time-integrated $B / \bar{B}$ mixing parameter. Other analyses have looked at lepton $+D_{s}$, lepton $+D^{0} / D^{ \pm}$and lepton $+\Lambda_{c}$ correlations to attempt to extract the species fractions. Once statistics have improved, the uncertainty in species fractions will be one of the dominant uncertainties in attempting to extract absolute branching ratios. 